\title{
Identification of Pharmacophoric features and novel compounds for inhibition of SARS-Cov-2 Main Protease
}

\author{
Priti Jain*a, Rupesh Dorik $^{\text {a }}$, Munendra Jain ${ }^{\mathrm{b}}$ \\ ${ }^{a}$ Department of Pharmaceutical Chemistry, R C Patel Institute of Pharmaceutical Education and \\ Research, Shirpur-Maharashtra (India) 425405 \\ ${ }^{b}$ School of Technology, SVKM's Narsee Monjee Institute of Management Studies, Indore Campus, \\ Madhya Pradesh-India
}

Corresponding author: pmj.grv@gmail.com

\begin{abstract}
A big race for the search for novel lead has begun due to the emergence of COVID-19 across the globe. More than 6,00,000 cases of afflicted patients worldwide has been reported till date with high mortality and morbidity. At present no approved drugs are known for COVID-19. Phylogenetic analysis present strong nucleotide sequence similarity of around $80 \%$ with SARS-CoV. Therefore, the drugs used for treating SARS-CoV and MERS are being used for SARS-CoV-2 also. Recently, the crystal structure of COVID-19 is reported and hence, we have used this tom predict the binding affinity with SARS-CoV-2-main protease and prepared a pharmacophore that may be used for future design of novel inhibitors.
\end{abstract}

Keywords: SARS-CoV-2, COVID-19, Protease, Docking, Pharmacophore, Zinc15

\section{Introduction:}

The end of year 2019 and beginning of 2020 came as a big human threat, the SARSCoV-2 or nCOVID-19. This pandemic has become a global health concern and is issue of economical, social and psychological burden. COVID-19 outbreak was first witnessed at Wuhan district of China in December 2019 and was recognized and declared a pandemic by World Health Organization on $11^{\text {th }}$ March 2020 [1]. Worldwide outbreak of the disease as of March 2020 has affected 202 countries and confirmed about 6,17,000 cases with 28,335 deaths and still counting [2]. Evidences of SARS CoV-2 being related to SARS have been proved by their phylogenetic studies and can be correlated for the research and development of novel antiviral drugs and vaccines. Coronavirus belongs to Nidivirales order and Coroaviridae family and contains nucleic material as single 
stranded RNA having size $26-32 \mathrm{kbs}$ in length. It has alpha $(\alpha), \operatorname{beta}(\beta), \operatorname{delta}(\delta)$ and $\operatorname{gamma}(\gamma)$ coronavirus subgroups, the $\alpha$ and $\beta$ subgroups have the ability to infect humans and are pathogenic. SARS and SARS CoV-2 belong to the $\beta$ coronavirus subgroup [3]. Peculiar feature in SARS coronavirus, MERS (Middle East respiratory syndrome) and SARS CoV-2, H1N1 and H5N1 influenza-A virus was their ability to infect human host leading to pulmonary disorders and gradually resulting in death. First incidence of this outbreak affecting humans and causing death was witnessed due to the SARS CoV (Severe acute respiratory syndrome coronavirus) in 2002, China and later due to the outbreak of MERS (Middle east respiratory syndrome) in middle east countries like Saudi Arabia [4]. It is the sixth time in history that WHO has announced a global health emergency and that is 2019-nCoV [5].

COVID-19/ SARS CoV-2 reported in Wuhan, China in December 2019 is the $\beta$ subgroup coronavirus consisting of single stranded RNA with 29,903bp [6]. Hitherto, no treatment or vaccine is available for combating this dreadful viral infection. Researchers are putting strong efforts to identify the targets that may be exploited for development of novel therapeutics. Recent paper published in Science reported the crystal structure of SARSCoV-2 main protease. SARS-CoV-2 main protease is also known as $\mathrm{M}^{\text {pro }}$ or $3 \mathrm{CL}^{\text {Pro }}$ and this protein is essential for the transcription/replication of the RNA [7]. Reports suggest that protease inhibitors can be used for the treatment, hence we chose to exploit the HIV protease inhibitors for search of novel hits that may serve as 3CLpro inhibitors.

In-silico studies were performed on the known HIV protease inhibitors (Darunavir, lopinavir, ritonavir, saquinavir, indonavir, tipranavir) (figure 1) to predict the binding mode of these drugs on SARS-CoV-2 main protease (PDB: 6LU7). This protease comprises of 3 domains I, II and III and the substrate binding cleft is embedded between domain I and II which possess an antiparallel beta strand comprising of His41 and Cys145 as catalytic dyad. Docking simulation studies displayed the key interactions of protease inhibitors with main protease. It was observed that all the drugs bind well with the active site and displayed strong hydrogen bonding interactions and Vander Waal interactions. The obtained data was used to generate a pharmacophore by superimposition of all ligands in order to govern newer molecules. The generated pharmacophore was 
applied to Zinc15 and required filters were used to generate the leads from a pool of 213,000,000 compounds. The database gave 7904 compounds in search and on applying exhaustive filters for oral bioavailability, toxicity, Gibbs free energy we succeeded in obtaining 25 hits. These were then docked and the interactions were predicted.

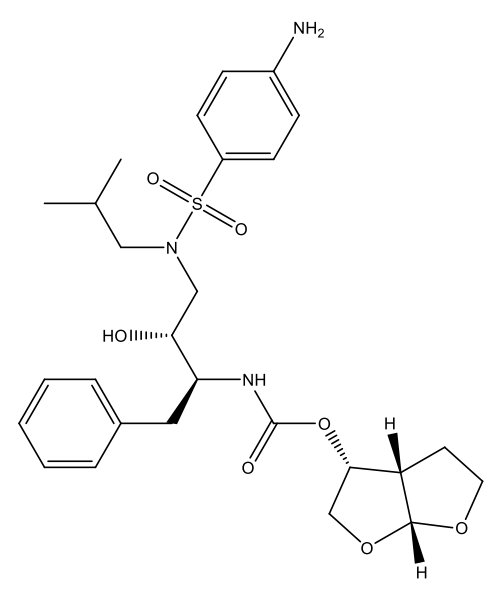

Darunavir

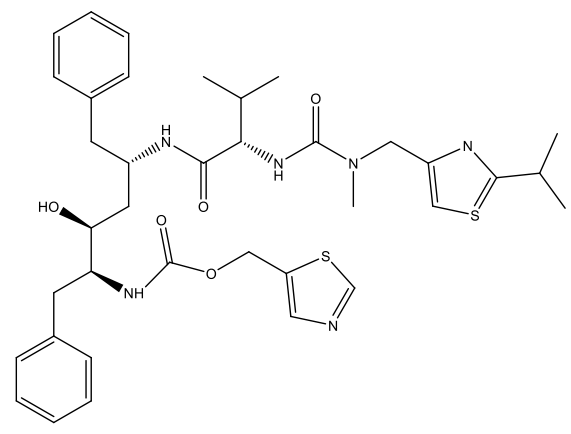

Ritonavir

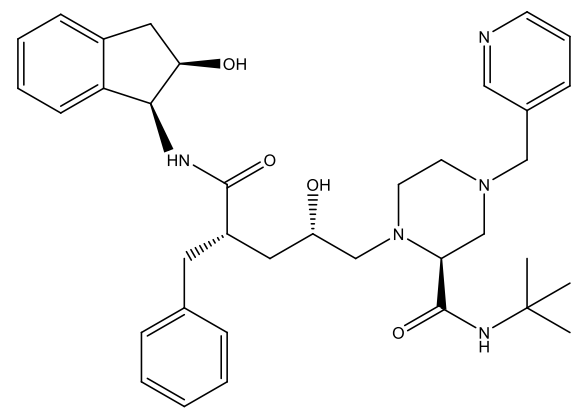

Indonavir

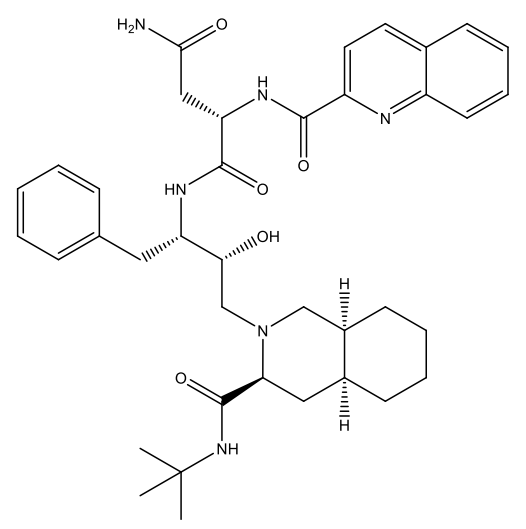

Saquinavir

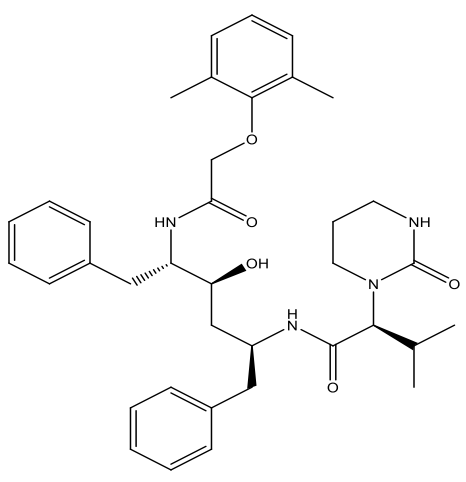

Lopinavir

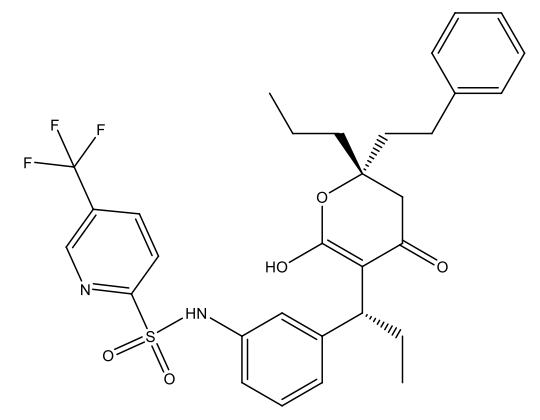

Tipranavir

Figure 1: Chemical structures of reported HV protease inhibitors

\section{Methodology:}

All the chemical structures were drawn using ChemDraw11.0 and saved as .cdx format. The docking simulation was performed using Swiss dock. Crystal structure of SARSCoV-2 main protease (6LU7) was downloaded from Brookhaven protein data bank and prepared using the preparation wizard of MVD. Protein was prepared for docking 
simulation by assigning missing bonds, bond orders, hybridization states, explicit hydrogen, charges and flexible torsion. The ligands to be docked were drawn using Chemdraw Ultra 11 and their 3D structure was energy minimized using MM2 (molecular mechanics 2) force field in Chem 3D Pro, version 11.0. All the energy minimized ligands were further prepared and corrected for bonds, bond orders, hybridization and charges through ligand preparation wizard. For the docking purpose, grid was generated around the co-crystallized ligand.. The system generates 5 possible energy minimized conformers for each ligand, which were visualized for interactions using ligand energy inspector and best pose was scored.

Pharmacophore generation was performed using Molegro virtual docker and Avagadro [8-9]. The results obtained were loaded on ZincPharmer to generate hits depending on the filters and pharmacophoric structure.

\section{Results and Discussion:}

The docking simulation of HIV protease inhibitors was performed on SARS-CoV-2 main protease. These gave excellent interaction with the active site residues which were observed through manual visualization. Secondary structure of protein shown in figure is clear depiction that docked ligands bind to the same site as co-crystallized compound (figure 3). The $-\mathrm{OH}$ group displayed hydrogen bonding interaction with Cys 145 and His164. Sulfonyl oxygen displayed strong hydrogen bonding with Gly143 while carbonyl oxygen interacted with Glu166 and 189. Further Vander Waal and steric interactions were observed with His 41, Thr25, Thr 26, Gly 143, Asn 142, Gln 189, Met 165. Moldock score was -162.191. The docked compounds were superimposed with the cocrystallized ligand; 02J-Ala-Val-Leu-Pje-010. Results were appealing as the docked compounds were seen to be present and extended interactions in the same manner as the peptidomimetic inhibitor does. Finding the results extraordinary, we tried to create a pharmacophore with existing HIV-protease inhibitors. The calculation of energy map in MVD revealed important amino acids with the kind of binding preferred by them. Figure given (5A) below represents a pharmacophoric model generated using MVD and PharmGist. Green colour represents favourable region for steric interactions, blue constraints favour the hydrogen acceptor and yellow favour the hydrogen bond donors. 
The figure represents all the possible iterations for active site residues but for further calculation we used the common features obtained from HIV protease inhibitors. Utilizing this information gave insight to screen library of large database ie ZINC15 using ZINCPharmer. Keeping Lipinsky rule of file as a search criteria, we were able to get 7,904 hits. On further screening with appropriate filters and selection strategies we sorted the best 25 hits. These were then subjected to docking simulation and the docking scores and interactions were determined. The compounds with exceptional high docking score are only presented in this paper.

ZINC00911333 interacted strongly with active site residues showing hydrogen bonding interactions with His 41, Ser144, Thr 25, Thr 45, Ser46 and steric interactions were seen with Cys145, Leu 141, Glu166, Thr24, and Thr45. It has excellent moldock score of 308.427 and energy accounting for hydrogen bond was greater than all other docked compounds (-5.601). Other hits obtained were Zinc06142910, Zinc08439206 from amongst the others (figure 2).

Table 1 given below presents the docking scores and binding affinities of HIV protease inhibitors and the best hit screened from ZINC database.

Table1: Docked compounds with moldock score and ligand efficiency

\begin{tabular}{|c|c|c|}
\hline Compound & Moldock score & Ligand Efficiency \\
\hline Darunavir & -166.907 & -4.099 \\
\hline Indinavir & -176.05 & -3.92 \\
\hline Ritonavir & -171.488 & -3.73 \\
\hline Saquinavir & -189.874 & -3.07 \\
\hline Tipranavir & -168.904 & -4.02 \\
\hline ZINC00911333 & -308.427 & -2.98 \\
\hline
\end{tabular}




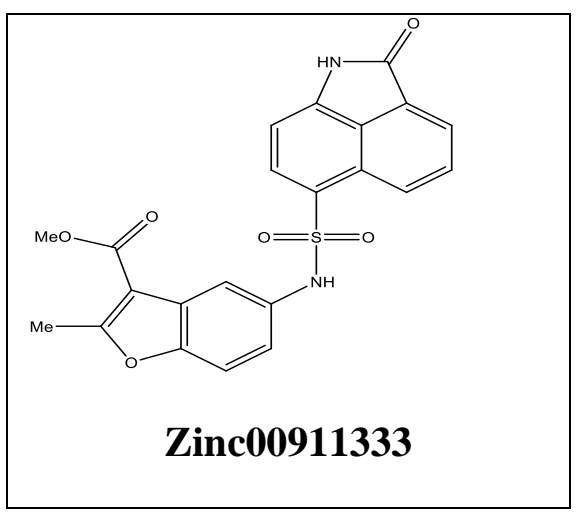

Figure 2: Chemical structure of the compound screened through Pharmacophoric search

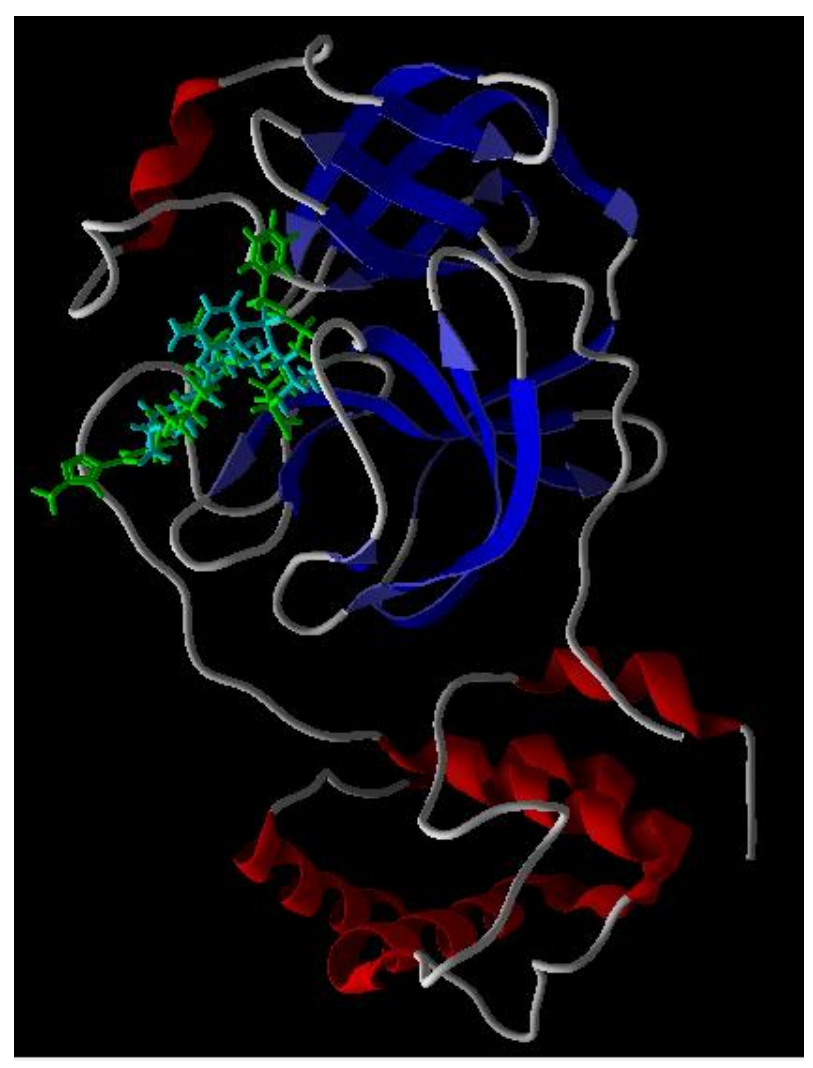

Figure 3: Ribbon structure of SARS-CoV-2 Main protease (6LU7) representing the domain I, II and III. The active site is represented with the co-crystallized peptidomimetic ligand (green) which is also seen to be well superimposed with Darunavir. 

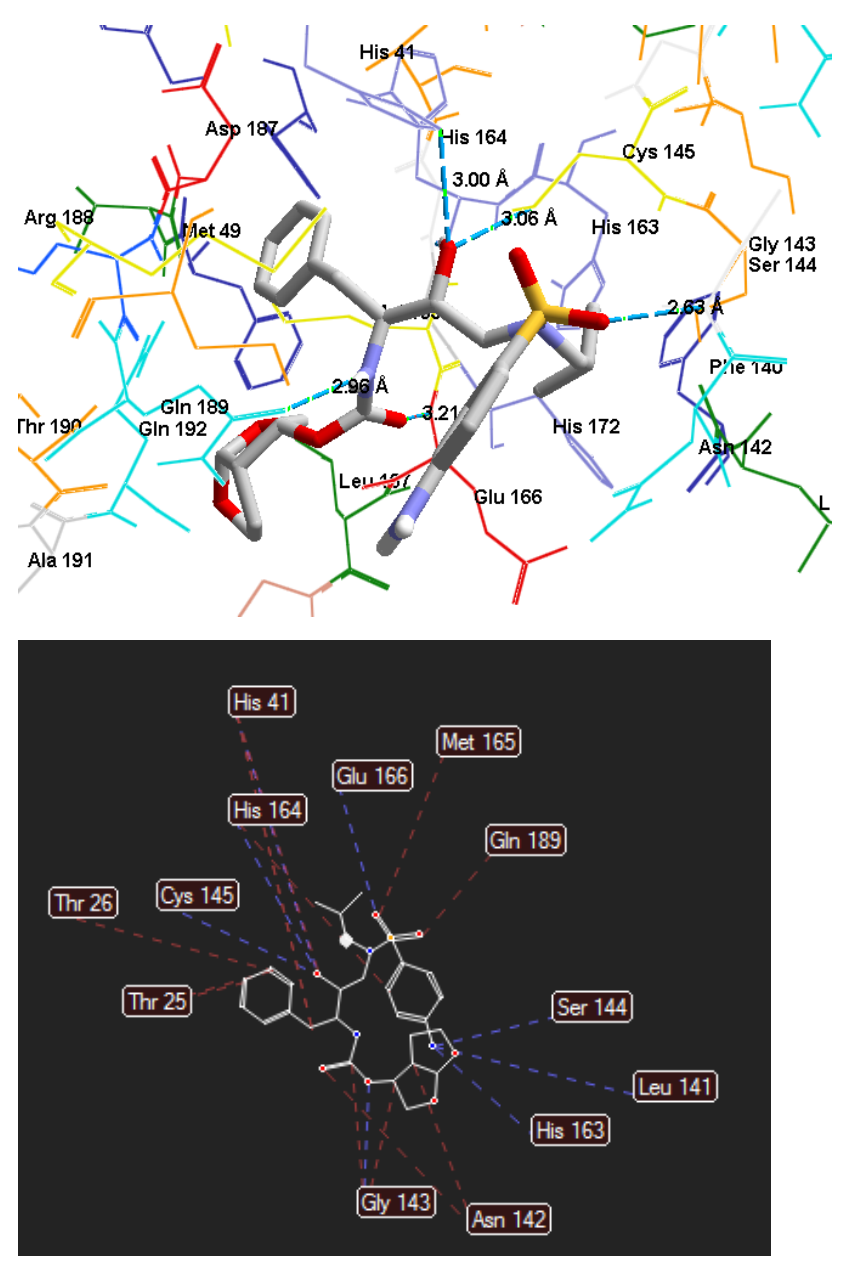

A

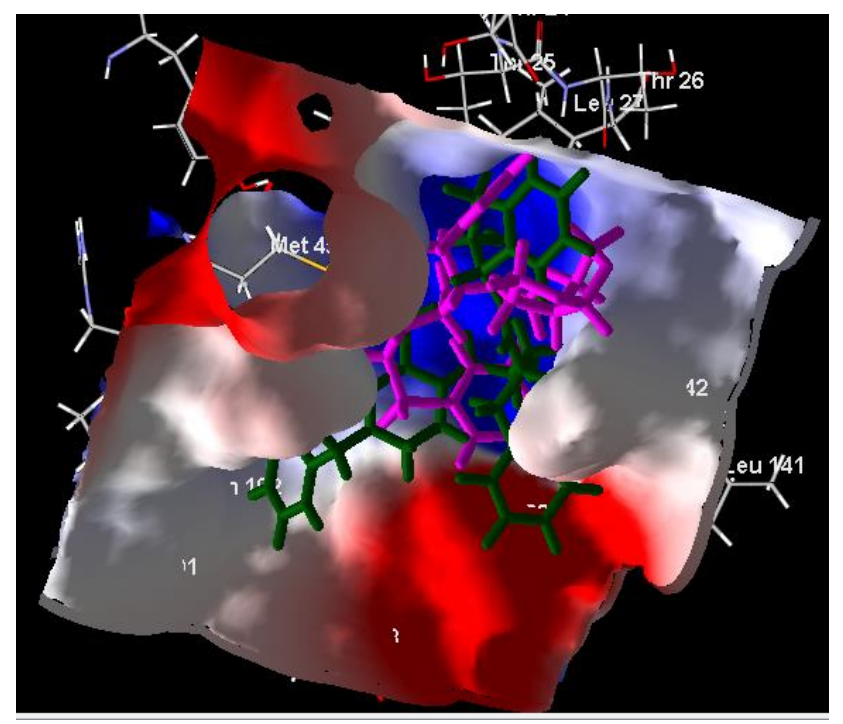




\section{C}

Figure 4: A: Docked pose of Darunavir in 6LU7; B: 2D interaction plot of Darunavir displaying hydrogen bonding interaction in blue and Vander Waals interaction in red.; $\mathbf{C}$ : The superimposed view of Darunavir (pink) and Indinavir (green)
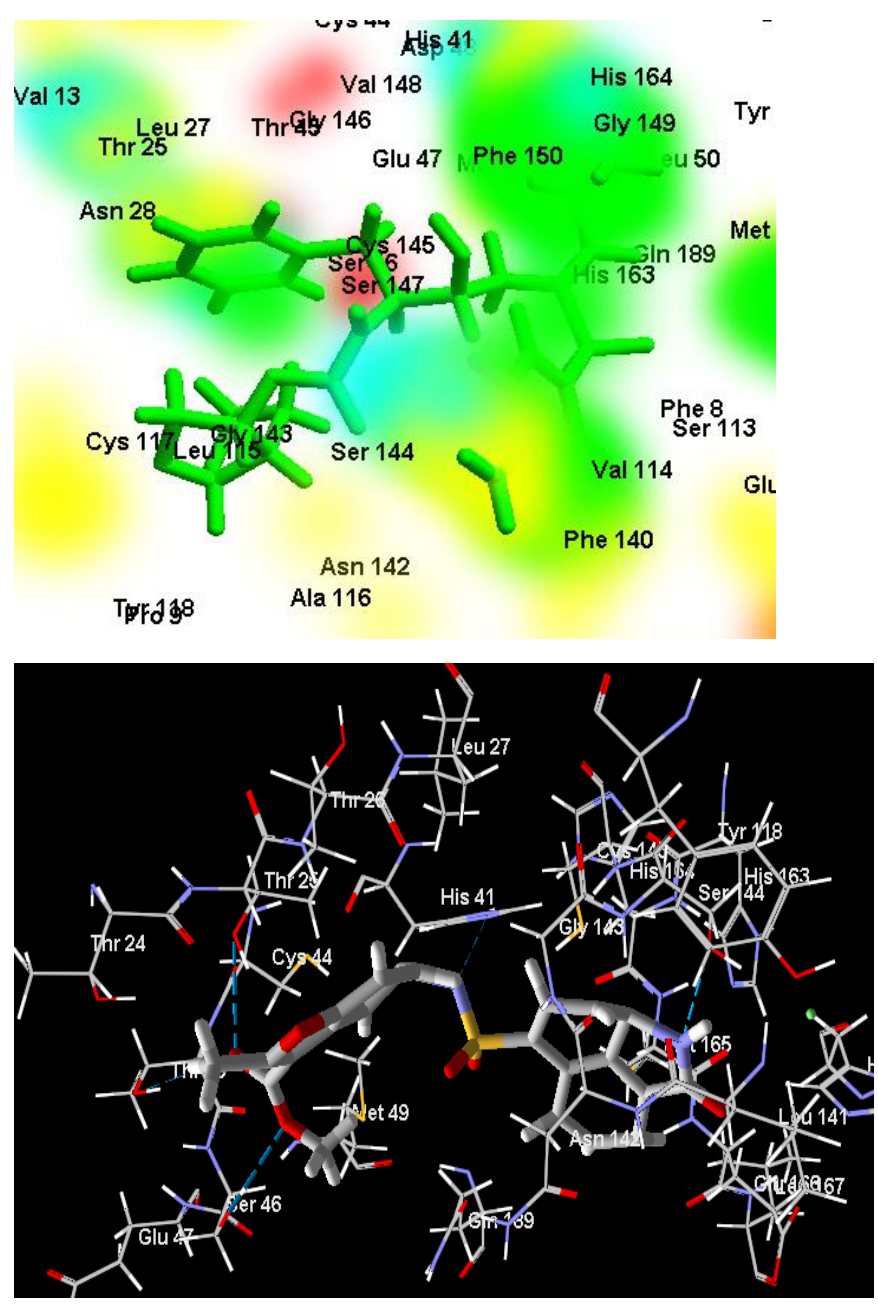

A 


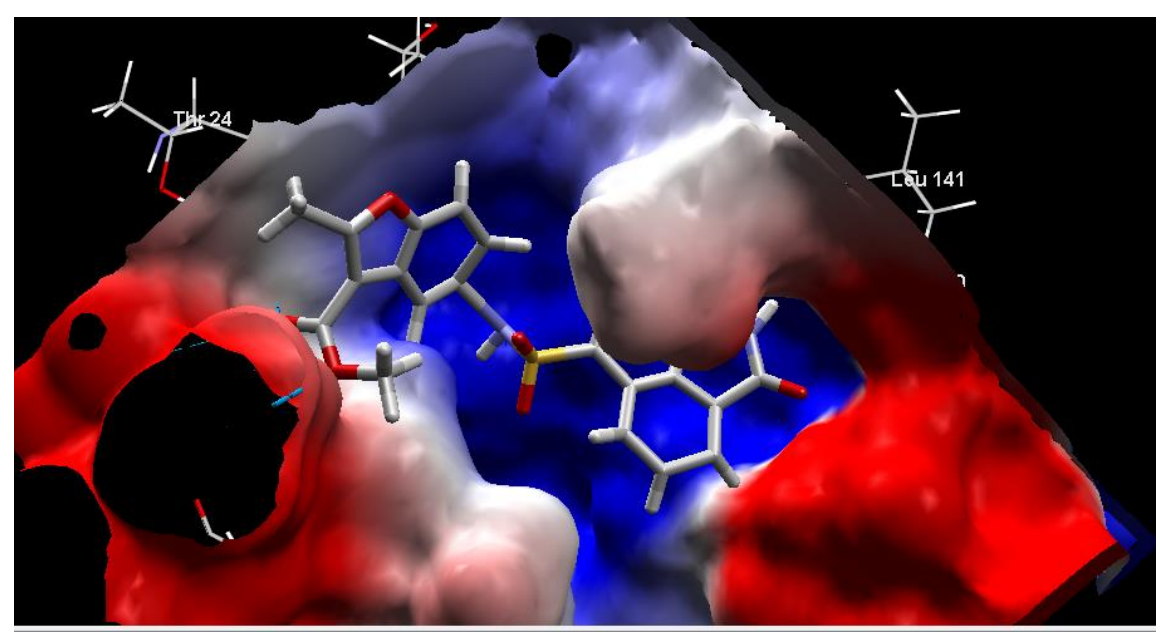

C

Figure 5: A: The figure depicting the pharmacophore generated using HIV protease inhibitors; $\mathbf{B}, \mathbf{C}$ : Docked pose for ZINC00911333

4. Conclusion: Considering the critical situation being faced by humanity in present scenario due to COVID-19 and unavailability of approved drugs poses a great difficulty. As per the reports, HIV-1 protease inhibitors may be used and henceforth these are being used as treatment options. We have therefore, tried to determine the possible interactions these can have with SARS-CoV-2 main protease. In lieu of the results obtained, pharmacophore is generated that will surely facilitate the research for the design of novel inhibitors. The ZINC15 database screening also provided us with hits which may be purchased or synthesized and further evaluated for in vitro and in vivo 3CLpro inhibition.

\section{References:}

1. https://www.who.int/emergencies/diseases/novel-coronavirus-2019/events-asthey-happen [[Accessed 15March2020]]

2. https://www.cdc.gov/coronavirus/2019-ncov/cases-updates/summary.html [[Accessed 17March 2020]

3. Yadi Zhou, Yuan Hou, Jiayu Shen et al. Network-based drug repurposing for novel coronavirus 2019-nCoV/SARS-CoV-2, Cell Discovery, 6(14) (2020) 
4. https://www.who.int/csr/sars/en/WHOconsensus.pdf. Consensus document on the epidemiology of severe acuterespiratory syndrome (SARS)

5. 2019-nCoV outbreak is an emergency of international concern. Accessed from "http://www.euro.who.int/en/health-topics/health-emergencies/internationalhealth-regulations/news/news/2020/2/2019-ncov-outbreak-is-an-emergency-ofinternational-concern"

6. Muhammad Adnan Shereena,b,1, Suliman Khana et al. COVID-19 infection: Origin, transmission, and characteristics of humancoronaviruses. Journal of Advanced Research. 24, 2020, 91-98

7. Linlin Zhang, Daizong Lin, Xinyuanyuan Sun. Crystal structure of SARS-CoV-2 main protease provides a basis for design of improved $\alpha$-ketoamide inhibitors. Science 20 Mar 2020: eabb3405

8. Avogadro: an open-source molecular builder and visualization tool. Version 1.XX. http://avogadro.cc/

9. Marcus D Hanwell, Donald E Curtis, David C Lonie, Tim Vandermeersch, Eva Zurek and Geoffrey R Hutchison; “Avogadro: An advanced semantic chemical editor, visualization, and analysis platform" Journal of Cheminformatics 2012, 4:17.

10. https://bioinfo3d.cs.tau.ac.il/PharmaGist/

11. Inbar Y, Schneidman-Duhovny D, Dror O, Nussinov R, Wolfson HJ. Deterministic Pharmacophore Detection via Multiple Flexible Alignment of Drug-Like Molecules. In Proc. of RECOMB 2007, vol. 3692 of Lecture Notes in Computer Science, 423-434. Springer Verlag.

12. Schneidman-Duhovny D, Dror O, Inbar Y, Nussinov R, Wolfson HJ. PharmaGist: a webserver for ligand-based pharmacophore detection. Nucleic Acids Research 2008

13. ZINCPharmer: pharmacophore search of the ZINC database , David Ryan Koes, Carlos J. Camacho Nucleic Acids Research, 2012, Vol. 40, Web Server issueW409-W414 
14. Sterling and Irwin. J. Chem. Inf. Model, 2015. http://pubs.acs.org/doi/abs/10.1021/acs.jcim.5b00559. 